is naturaleza 


\section{DOSSIER CRÍTICO SOBRE EL LIBRO:}

\section{LOS SÓTANOS DEL UNIVERSO}

Volumen monográfico de

\section{NATURALEZA Y LIBERTAD}

Revista de estudios interdisciplinares

Número 3

Málaga, 2014

Esta revista es accesible on-line en el siguiente portal: http://grupo.us.es/naturalezayl 


\section{Naturaleza y Libertad}

Revista de estudios interdisciplinares

Número 3, 2014

ISSN: 2254-96682014

Directores: Juan Arana, Universidad de Sevilla; Juan José Padial, Universidad de Málaga; Francisco Rodríguez Valls, Universidad de Sevilla.

Secretaria: Avelina Cecilia Lafuente, Universidad de Sevilla.

Consejo de Redacción: José Luis González Quirós, Universidad Juan Carlos I, Madrid; Francisco Soler, Universität Dortmund / Universidad de Sevilla; Pedro Jesús Teruel. Universidad CEU Cardenal Herrera; Héctor Velázquez, Universidad Panamericana, México.

Adjunto a la redacción: Miguel Palomo, Universidad de Sevilla

Consejo Editorial: Mariano Álvarez, Real Academia de Ciencia Morales y Políticas; Allan Franklin, University of Colorado; Michael Heller, Universidad Pontificia de Cracovia; Manfred Stöcker, Universität Bremen; William Stoeger, University of Arizona.

Consejo Asesor: Rafael Andrés Alemañ Berenguer. Universidad de Alicante; Juan Ramón Álvarez, Universidad de León; Luciano Espinosa, Universidad de Salamanca; Miguel Espinoza, Université de Strasbourg; Juan A. García González, Universidad de Málaga; José Manuel Giménez Amaya, Universidad de Navarra; Karim Gherab Martín, Urbana University, Illinois; Martín López Corredoira, Instituto de Astrofísica de Canarias; Alfredo Marcos, Universidad de Valladolid; Javier Monserrat, Universidad Autónoma de Madrid; Leopoldo Prieto, Colegio Mayor San Pablo, Madrid; Ana Rioja, Universidad Complutense, Madrid. Madrid; José Luis González Recio, Universidad Complutense, Madrid; Javier Serrano, TEC Monterrey (México); Hugo Viciana, Université Paris I; Claudia Vanney, Universidad Austral, Buenos Aires; José Domingo Vilaplana, Huelva.

\section{Redacción y Secretaría:}

Naturaleza y Libertad. Revista de estudios interdisciplinares. Departamento de Filosofía y Lógica. Calle Camilo José Cela s.n. E-41018 Sevilla.

Depósito Legal: MA2112-2012

용 954.55.77.57 Fax: 954.55.16.78. E-mail: jarana@us.es 
Naturaleza y Libertad. Revista de estudios interdisciplinares. Número 3, 2014. ISSN: 2254-9668

\section{ÍNDICE}

PRESENTACIÓN.

DOSSIER CRÍTICO SOBRE EL LIBRO LOS SÓTANOS DEL UNIVERSO

Juan Arana (U. de Sevilla), El problema de la determinación y el diálogo interdisciplinar......11

Miguel Acosta (U. CEU San Pablo, Madrid), La epistemología del riesgo y la

relación ciencia-filosofía. . .25

Carlos del Ama (Madrid), Reflexiones con ocasión del libro Los sótanos del universo ...........57

Luciano Espinosa (U. de Salamanca), De los sótanos al ático del universo ............................93

Gonzalo Génova (U. Carlos III, Madrid), Turing y el rostro del universo ..........................101

Julio A. Gonzalo (U. Autónoma de Madrid), De los sótanos a los confines del universo ....111

Juan Meléndez Sánchez (U. Carlos III, Madrid), De riesgos y metáforas

Andrés Muñoz Machado (Universidad Politécnica de Madrid), Las matemáticas en

Los sótanos del universo

Javier Sánchez Cañizares (U. de Navarra), Sótanos del universo, espejos del alma

Francisco José Soler Gil (U. de Sevilla), Una filosofía de la naturaleza contracorriente y antisistema.

Ignacio Sols (U. Complutense, Madrid), Recuperando la causa formal sin abandonar la causa eficiente. .151

Juan Arana (U. de Sevilla), Los subsótanos del universo. Materiales para un debate. 167 
Naturaleza y Libertad. Revista de estudios interdisciplinares. Número 3, 2014. ISSN: 2254-9668

\title{
LA EPISTEMOLOGÍA DEL RIESGO Y LA RELACIÓN CIENCIA-FILOSOFÍA
}

\author{
Miguel Acosta ${ }^{1}$ \\ Universidad CEU San Pablo
}

\begin{abstract}
Resumen: Los sótanos del universo plantea la necesidad de cambiar la epistemología del conocimiento apodíctico científico-filosófico basado en la certidumbre por la "epistemología del riesgo". Asimismo, sugiere un mayor acercamiento entre ciencia y filosofía para superar la actual confusión y estancamiento que afectan a estos saberes. Este artículo analiza ambos temas.
\end{abstract}

Palabras clave: ciencia, filosofía, epistemología, interdisciplinariedad.

Abstract: Los Sótanos del Universo proposes the necessity of changing the epistemology of apodictic scientific-philosophical knowledge, based on certainty, for the "epistemology of risk". Furthermore, it suggests a better approach between philosophy and science in order to surpass the confusion and standstill that affect these disciplines today. This paper analyses both matters.

Keywords: Science, Philosophy, Epistemology, Interdisciplinarity.

Recibido: 15 de enero de 2013. Aprobado: 12 de diciembre de 2013.

\section{INTRODUCCIÓN}

He podido comprobar que Juan Arana lleva razón cuando dice que su libro Los sótanos del universo es una síntesis de su pensamiento acerca de la

1 Profesor Adjunto de Filosofía de la Universidad CEU San Pablo y del Instituto CEU de Humanidades Ángel Ayala. 
Filosofía de la Naturaleza y la Filosofía e Historia de la Ciencia. Hace varios años atrás sigo sus trabajos porque coincido con él en muchas de sus tesis y me siento atraído por su apertura de pensamiento cuando aborda los temas más controvertidos sin temores ni compromisos. Admiro mucho esa capacidad de hablar sin pelos en la lengua, con la modestia de admitir las propias limitaciones, y al mismo tiempo, con el rigor que siempre ha caracterizado sus investigaciones. Me parece de una honestidad intelectual difícil de encontrar en nuestra cultura académica donde sobre todo impera lo "políticamente correcto". Así pues, con mucho gusto y sin mérito alguno por mi parte, he aceptado la invitación de Fernando Fernández para colaborar en los comentarios de este último libro del Prof. Arana.

En mi intervención durante el encuentro que hemos tenido en el Seminario Bibliográfico de AEDOS no pude exponer de manera ordenada uno de los temas que aparecen de manera subyacente en Los sótanos del universo, aunque es mencionado de forma explícita en varias partes de esta obra. Me refiero a la relación entre Ciencia y Filosofía. En efecto, el libro comienza presentando el problema epistemológico y metodológico de la actual Filosofía de la Naturaleza y luego trata sintéticamente un amplio espectro de cuestiones muy actuales: el azar y las causas, las leyes y la complejidad, la vida y el determinismo, la matemática y la metafísica, entre otros.

Desde el primer momento, cuando se plantea la cuestión metodológica, nuestro autor propone una "epistemología del riesgo" donde, a mi modo de ver, apela a una revisión de las "intenciones epistemológicas" de la ciencia moderna y de la filosofía para dejar a un lado el paradigmático ideal cartesiano-kantiano de la "certeza del conocimiento". A continuación intentaré explicar más detenidamente lo que pretendo decir con esto, y sobre todo, 26 
procuraré justificar la importancia que tiene la aproximación y colaboración entre científicos y filósofos en la búsqueda de explicaciones legítimas de la realidad. Para ello me apoyaré algunos argumentos de Arana sobre todo de Los sótanos del universo y también añadiré algunas ideas propias que me han inspirado sus obras.

Una de las tesis fundamentales de Juan Arana es la necesidad de superar la dicotomía Ciencia-Filosofía y trabajar en colaboración. En realidad las fronteras entre una y otra no son tan marcadas como ha pretendido imponer el modelo científico-experimental surgido en el Renacimiento que buscaba ante todo certidumbre o conocimiento apodíctico. Arana, en cambio, defiende lo que denomina "epistemología del riesgo".

\section{UN POCO DE HISTORIA SOBRE EL MÉTODO}

Es lugar común admitir que en el siglo XVII ha habido un punto de inflexión en la historia del pensamiento científico occidental. Sin entrar en el debate de autores, obras o descubrimientos que han sido los principales agentes causales de este cambio que llevó siglos, las propuestas de Francis Bacon en el Novum Organum Scientiarum (1620) y de René Descartes en el Discours de la méthode pour bien conduire la raison et chercher la vérité dans les sciènces (1637) acerca de una nueva forma de "asegurar" el conocimiento científico auténtico y verdadero darían lugar al nacimiento de la Ciencia Moderna. La aplicación del nuevo método resultó muy significativa en los trabajos de Galileo y de Newton que con su Philosophiae Naturalis Principia Mathematica (1687) remata y finaliza la conocida Revolución Científica. De esta forma, la inducción y la deducción, la experimentación y la fundamenta- 
ción matemática serían los pilares fundamentales de la nueva ciencia. Su efectividad estaba demostrada y el entusiasmo ante el definitivo "progreso continuo" ya no se detendría.

Arana critica el abuso dado al término "revolución" aplicado a numerosos descubrimientos científicos en los últimos siglos. En su obra El caos del conocimiento. Del árbol de las ciencias a la maraña del saber, dice que, sin negar que en sentido limitado ha habido varias revoluciones científicas,

...el carácter genuinamente revolucionario de la ciencia misma procede de la unidad profunda de la transformación introducida en el estudio de la naturaleza a partir del Renacimiento. Mi tesis es que todas las revoluciones que ha conocido la ciencia desde Copérnico hasta Einstein, Niels Bohr o Francis Crick, forman parte de una sola revolución, por medio de la cual se dio la vuelta por completo, pero una sola vez, al modelo de conocimiento que había regido hasta ese momento ${ }^{2}$.

No cabe duda de que el modelo establecido ha funcionado en el sentido de que ha encontrado verdades causales para explicar numerosos efectos de la naturaleza y, hasta cierto punto, controlarlos o al menos conducirlos. Se han aclarado incógnitas sobre nuestra situación en el cosmos y en nuestra biosfera, a la vez que se han solucionado problemas que afligieron a la humanidad durante muchos siglos en cuanto a salud, medios de comunicación, transporte y alimentación, entre otros. Desde la adopción del nuevo modelo, la ciencia moderna no ha dejado de sorprendernos con nuevos des- 
cubrimientos. No resulta extraño comprender la confianza de los intelectuales de los siglos XVIII y XIX al decir que se lograría un progreso continuo que conduciría a la felicidad y el bienestar de todos los hombres. En especial cuando dichos descubrimientos se concretaban en resultados tecnológicos que procuraban una vida más llevadera y grata. ¿Era necesario seguir contando con la vieja filosofía?

La filosofía no era ajena a todo este proceso de cambio, sobre todo porque se iba produciendo en su mismo seno. Ciencia y filosofía constituían un único saber desde la antigüedad griega. No existía la fuerte "demarcación epistemológica" que se observa actualmente ${ }^{3}$. Pitágoras, Platón, Aristóteles, Arquímedes o Alberto Magno se dedicaban a todas las ramas del saber en sus respectivas épocas. Ahora bien, cuando en la Edad Moderna comienzan a aumentar los conocimientos y el nuevo modelo exige una mayor dedicación para la experimentación y verificación, se lleva a cabo de forma paulatina la "especialización". La división de áreas científicas se produjo de forma práctica, aunque teóricamente los "científicos" eran todavía filósofos, o al menos "naturalistas". Kepler, Galileo, Descartes, Leibniz, Newton... fueron hombres de ciencia y de filosofía (basta con recordar el título de la principal obra de Newton). Se dedicaban a la Filosofía de la Naturaleza, eran "naturalistas" como lo serían también Lavoisier, Huygens, Linneo o Darwin. En el siglo XIX la especialización se produce de manera más formal y la ciencia se

3 Arana señala esta situación en un comentario a la Historia General de la Naturaleza de Kant. Cf. J. Arana, Ciencia y metafísica en el Kant precrítico: 1746-1764, Sevilla, Secretariado de Publicaciones de la Universidad de Sevilla, 1982, pp. 60-61. 
pluraliza continuamente clasificándose y sub-clasificándose en distintas ramas tal como se la conoce hoy día.

Sin embargo, la realidad percibida es que a medida que la ciencia moderna ha ido aumentando en éxitos y prestigio, la filosofía ha ido menguando hasta convertirse en una especie de baúl de los recuerdos. Así, las relaciones entre filosofía y ciencia moderna han sido tensas y se han ido resquebrajando hasta producirse un claro distanciamiento. El hijo ha crecido tanto que vio a su padre completamente prescindible, pensó que ya nada le podía aportar porque le resultaba anacrónico e insatisfactorio, e incluso intentó sustituirle en algunas de sus funciones específicas. En este sentido, Arana recuerda el cuento de Cortázar, La casa tomada:

Aquí la metáfora de la casa tomada no es la que mejor cuadra, sino en todo caso la de casa abandonada por cese del negocio. De todos modos, el final es el mismo y la llave también va a parar a la alcantarilla. [...] ¿ ¿A] qué se dedicarán los filósofos después del desahucio? $\mathrm{Su}$ opción preferida ha sido realojarse en casas ajenas a cambio de sus servicios. Es frecuente que pretendan convertirse en guardias de tránsito para regular la circulación del conocimiento, o garantes del buen uso del lenguaje, o ministros sin cartera que ejercitan por doquier el "espíritu crítico". Funciones no demasiado apreciadas por sus presumibles beneficiarios, lo que explica el escaso entusiasmo con que han sido recibidos en todas partes estos filósofos "desterrados". Es comprensible que la gente prefiera efectuar por sí misma las funciones crítico-reflexivas, de manera que los que esperaban ejercerlas por cuenta ajena acaban viéndose sin oficio ni beneficio. ${ }^{4}$

4 J. Arana, Los sótanos del universo. La determinación natural y sus mecanismos ocultos, Madrid, Biblioteca Nueva, 2012, p. 44. Los subrayados son del autor. 
Es muy duro el dictamen y, dentro de la misma Filosofía, la metafísica es la que más palos ha recibido. ¿Cómo se llegó a este punto? Gran parte de la culpa es de la misma Filosofía ya que desde su seno avaló el nuevo modelo "a cualquier precio". Arana dice que uno de los principales promotores de esta situación fue Kant ${ }^{5}$. Señala de qué manera la certidumbre y la evidencia propuestas por Descartes se convierten en sucedáneos de la verdad. Es decir, se intenta asumir la fórmula: "verdad = certeza $=$ evidencia" 6 . En relación a la verdad, Kant tuvo la oportunidad de decantarse por la certeza (subjetiva) o por la evidencia (objetiva) y "[p]or desgracia para todos, el filósofo alemán hizo la peor elección posible, porque interpretó la verdad que creyó hallar en la física newtoniana con una clave que tomó prestada de la metafísica racionalista"7, quiso quedarse con ambas a la vez. Tan seguro estaba de lo

5 En su obra Ciencia y Metafísica en el Kant precritico: 1746-1764 anteriormente mencionada, Arana detecta la raíz del problema kantiano que se pondrá claramente de manifiesto en las Críticas. Pero también acusa la rigidez de algunos filósofos aristotélicos al tratar de encontrar conceptos que se refieren de manera exacta a algunas realidades que no se dejan atrapar en ellos. Por ejemplo, el tema de la causalidad y la metafísica: cf. p. 345.

6 Ídem, p. 37. Como sabemos, en la filosofía clásica estos términos no son sinónimos. La verdad en sentido ontológico se refiere a la realidad misma, que sería un trascendental; y la verdad lógica apunta a una relación entre el conocimiento del sujeto y la realidad fuera de él. En este caso se trataría de la verdad ontológica. La certeza es lo subjetivo. Es una disposición del sujeto que muestra una seguridad de estar ante una verdad que suele suceder al encontrarse con una evidencia (a veces se habla de grados de certeza: duda, conjetura, opinión). La evidencia es lo objetivo, aquello que podemos percibir mediante la representación y que constituye una señal que avala aquello que es en la realidad y por tanto conduce a la verdad. Así, la certeza es la convicción firme de estar ante una verdad porque se basa en una evidencia. 7 Ídem, p. 35. 
definitivo de los descubrimientos de Newton que minusvaloró la utilidad de las hipótesis ${ }^{8}$. En otras palabras, consideró que ni siquiera se podía opinar acerca de las certezas, que había que huir de todo lo que se pareciera a una hipótesis o conjetura para ir por el camino seguro de la ciencia. Este planteamiento sólo fue posible desde el racionalismo inmanentista. Lo que Kant consiguió fue dividir el saber en ciencias abiertas (con un conjunto inacabable de objetos) y ciencias cerradas (la razón sólo se ocupa de sí misma tanto de la construcción formal como de las condiciones de posibilidad para constituir los objetos de la intuición sensible y de los conceptos del entendimiento) ${ }^{9}$. "Así pues Kant, para preservar el alto estándar de rigor que ha establecido, tiene que redimensionar la metafísica y transformarla en un saber propedéutico al estilo de la lógica. Se supone que así salvará al menos la ciencia natural de las dudas escépticas planteadas por críticos radicales como Hume."10 Pero Kant con su método consigue negar la posibilidad del tan anhelado conocimiento apodíctico a ciencias como la química o la biología. Al final se ve obligado a admitir que a la postre "ni la ciencia es tan segura ni la filosofía $\tan$ incierta"11.

De cualquier manera, aunque su objetivo de alcanzar una fundamentación adecuada para obtener el conocimiento apodíctico resultara un fracaso, la crítica kantiana favoreció más a las ciencias naturales que a la filosofía. Los

8 Conocimiento que no le había llegado estudiando a Newton directamente, sino a partir de las enseñanzas filosóficas de Christian Wolff.

9 Cf. J. Arana, Los sótanos del universo..., op.cit., p. 37.

10 Ibidem.

11 Ídem, p. 38. 
científicos pasaron por alto las complejas argumentaciones de Kant y mantuvieron sus conquistas en el terreno práctico y técnico, buscando en ellas la tan deseada certeza. Los filósofos lo tuvieron más crudo. Algunos decidieron acotar los límites para tratar sólo aquellos problemas que pudieran resolverse con el grado de certeza que se exige a una ciencia "digna de tal nombre" y poner entre paréntesis los temas inabordables o carentes de sentido. Otros subrayaron que el cometido del filósofo consiste en reflexionar, completando algunas argumentaciones que las ciencias positivas no cerraban como se debía. La tercera variante que comenta Arana fue la de encontrar un nuevo método para la filosofía de tal manera que pudiera recuperar la credibilidad científica, como es el caso de la fenomenología de Husserl.12

\footnotetext{
Obtener un criterio epistemológico de demarcación infalible, crear un lenguaje sin sombra de imprecisión, o efectuar una reducción fenomenológica perfecta no es menos difícil que detectar los límites del universo o asegurar la primacía del ser sobre la nada. En definitiva, cuando uno se empecina en la vía del rigor lo que se obtiene no es ciencia ni tampoco filosofía, sino un fracaso a veces épico y otras penoso. El caso se ha repetido tantas veces que ha desembocado por último en un proceso de autodestrucción de la filosofía, porque sus representantes oficiales renuncian a la identidad histórica de la disciplina para correr en pos de un imposible. No existe un más allá de Hume: el único desenlace honrado tanto del racionalismo explícito como del criptorracionalismo empirista es la impotencia escéptica. La clave del éxito de las ciencias positivas es que han sabido ser ciencias sin ser estrictas. Cualquier proyecto filosófico que pretenda otra cosa lleva en su dotación genética el germen de la muerte precoz. ${ }^{13}$
}

12 Cf. Ídem, pp. 41-42.

13 Ídem, pp. 42-43. E1 subrayado es de la fuente. 
La historia no acaba aquí, esto es lo que parece imperar en el ambiente intelectual de nuestros tiempos: el auge de la ciencia moderna y el deceso de la filosofía. Sin embargo, - gracias a Dios hay un "sin embargo" para los que hemos optado por dedicarnos a la filosofía - esta percepción que tiene gran parte de verdad comienza a cambiar. Nuevos vientos se han levantado en las últimas décadas y el siglo XXI exige nuevas condiciones de investigación. Veamos dos signos interesantes que han comenzado a aparecer ya en el siglo $\mathrm{XX}$, el primero con relación al conocimiento humano y el segundo con respecto a las crisis globales.

\section{EL CAOS DEL CONOCIMIENTO Y EL FANTASMA DE LA INTERDISCIPLINARIEDAD}

El triunfalismo con el que los científicos inauguraron el siglo XX fue rápidamente acallado nada más empezar esa centuria. Voy a mencionar dos aspectos que a mi modo de ver son importantes para el cambio epistemológico que se produce en el siglo XX: 1) La tesis de la certeza de las respuestas científicas, y 2) la tesis del progreso continuo de la humanidad mediante el avance científico. Ambas, que parecían haberse demostrado suficientemente durante la modernidad, se estrellaron estrepitosamente. Vamos a por la primera tesis.

El siglo XIX fue tan espectacular en cuanto a los avances científicos y nuevos descubrimientos que se respiraba un aire victorioso y optimista hacia finales de ese siglo. Es conocido el discurso de Sir William Thomson — Lord Kelvin — del 27 de abril de 1900 en la Royal Society de Londres bajo el título 
"Las nubes del siglo XIX sobre la Teoría Dinámica del Calor y la Luz"14. En dicho discurso dijo que la física era un conjunto perfectamente armonioso y - en lo esencial- acabado en el que solamente veía dos pequeñas nubes oscuras: el resultado negativo de la experiencia de Michelson y Morley, y la catástrofe ultravioleta de la ley Rayleigh-Jeans ${ }^{15}$. Para disipar tales nubes hizo falta cuestionar todo lo que hasta el momento se sabía del espacio, tiempo, masa y movimiento, en definitiva de la supuestamente inconmovible Física Mecánica Newtoniana. La solución se presentó de la mano de la Teoría de la Relatividad y de la Teoría de los Cuantos propuestas por Einstein y Planck respectivamente.

Admitir que la Física clásica era insuficiente para explicar aspectos fundamentales de la realidad natural fue un duro golpe para la ciencia moderna, pero también cabe decir que ella supo recibirlo y superarlo, de ahí las solu-

14 Cf. www.books.google.es (Última visita: 9 Enero 2013): W. Thompson, "NineteenthCentury Clouds over the Dynamical Theory of Heat and Light" en: W. Nicol (Ed.), Notices of the Proceedings at the Meetings of the Members of the Royal Institution, with Abstracts of the Discourses, Vol.16, Royal Institution of Great Britain, 1902, pp. 303 y ss.

15 Recordemos que este experimento óptico intentaba determinar la velocidad de la tierra con respecto al éter del universo. La presencia del éter (fluido sutil) como sustento de los astros o como medio propagador de la luz en el universo fue una de las teorías antiguas que pervivieron durante siglos formando parte del saber científico, así como la generación espontánea, la piedra filosofal o el fijismo de las especies. Por su parte, la "catástrofe ultravioleta" fue la expresión utilizada por los físicos del siglo XIX para referirse al extraño comportamiento de las ondas electromagnéticas en el estudio del "cuerpo negro" (constructo teórico para calcular las longitudes de onda y las intensidades de las diferentes radiaciones de que está compuesto un cuerpo que emite luz cuando experimenta calor). La teoría clásica no podía explicar la curva teórica de las pequeñas longitudes de onda (zona ultravioleta del espectro lumínico). 
ciones obtenidas para estos dos problemas. Ante la frase de Lord Kelvin uno se pregunta "de qué hay que asombrarse más, si del candor del sabio que creía en el fin de la Ciencia o de la agudeza de un espíritu capaz, incluso sin saberlo, de ver exactamente de dónde vendría la tempestad" 16 .

La filosofía no puede jactarse de esta "derrota" científica porque el golpe también le afectó y aceleró la caída del paradigma racionalista imperante en esa época. Aunque el movimiento romántico de finales del siglo XVII y principios del XVIII se mantuviera latente, la fuerza de la filosofía idealista tuvo preeminencia especialmente con la filosofía hegeliana. Pero a principios del siglo XX es ampliamente criticado y surgen nuevas propuestas como la fenomenología, la filosofía analítica o la hermenéutica. Las nuevas propuestas filosóficas se vieron influidas por la tragedia de las dos guerras mundiales que reformularon las cuestiones sobre la existencia humana y su dignidad (existencialismos y personalismos). Asimismo, la filosofía asume el papel de juez y comienza su labor crítica analizando la situación de las ciencias positivas. Entonces se proponen las teorías epistemológicas de Popper, Kuhn, Lakatos, entre otros, que cuestionan y revisan las bases metodológicas del modelo científico positivo.

La etapa del deslumbramiento por el modelo científico que se había experimentado sobre todo en el siglo XIX dio paso a una actitud más prudente de análisis crítico. El fantasma del escepticismo se hizo presente y aunque la pretensión de certeza luchó por mantenerse, el bote que conduciría a la felicidad universal gracias al progreso continuo de la ciencia comenzó a 
hacer agua por varias partes y empezó a cundir el pánico. El modelo se había topado de nuevo con la incertidumbre que llevaría al caos del conocimiento. Una vez más no se podía tener la certeza deseada en los resultados de la ciencia. La salida fue parapetarse insistiendo en la especialización de cada disciplina para afinar las respuestas y salvar la situación. Se realizaron ajustes metodológicos que más que certidumbre teórica procuraban efectividad práctica y, para llenar algunos huecos que dejó la filosofía, se consolidaron los métodos cualitativos de las ciencias sociales. De cualquier manera, en todas estas disciplinas continuó un hecho común: la especialización. Quisiera detenerme en este hecho.

El desarrollo de la ciencia moderna dio lugar a la sistematización del lenguaje científico, surgieron nuevas formas de nomenclatura, métodos de investigación y procedimientos que se especificaron en cada disciplina. Esto hizo que los científicos en sus respectivos campos pudieran concentrarse en los problemas que surgían y resolverlos sin "distraerse" con los problemas de otras disciplinas. La especialización fue sumamente eficaz a la hora de encontrar respuestas a problemas concretos, lo que ayudó a mejorar las aplicaciones prácticas y avanzar en los conocimientos de cada área. Por ejemplo, las reformas propuestas por Lavoisier en Química tuvieron una importancia estratégica en el proceso de madurez de esta ciencia: se concibió una nueva terminología y nomenclatura de los elementos, se definieron los métodos de investigación en el laboratorio y se realizaron mejoras técnicas que impulsaron su desarrollo. Gracias a eso se pudo llevar a cabo el estudio de los gases - la teoría del flogisto sucumbió definitivamente- y mejoró la comprensión de la termodinámica. 
Sin embargo, el precio de la especialización fue el aislamiento de cada disciplina con respecto a otras áreas "emparentadas" o "vecinas". Los científicos comenzaron a concentrarse en aspectos cada vez más puntuales y específicos de su área y los nuevos descubrimientos aumentaron rápidamente. No había tiempo para estar en todos los frentes, hasta que llegó un momento en el que empezó a producirse un distanciamiento tal que fue difícil la comunicación. Esta sigue siendo la situación científica actual, que por cierto también se extiende a las disciplinas humanísticas. Arana cuenta una experiencia de esta "incomunicación":

...por una serie de circunstancias me he sentado a conversar sobre el particular con físicos, biólogos, teólogos, economistas, ingenieros y estudiosos de la literatura. Lo único que contrapesó en todos los casos la certeza de no ser mínimamente entendido por ellos fue la sospecha de que a mi vez no conseguía —o tal vez no lo intentaba con la seriedad requerida - entenderles a ellos. Puesto a ser pesimista diría que es posible que estemos viviendo un segundo episodio de la afamada torre de Babel. ${ }^{17}$

Las consecuencias de la especialización son favorables en un aspecto pero peligrosas en otros ya que al intentar explicar un hecho de la realidad se puede caer en el error que narra el cuento indio de los seis ciegos y el elefante. Cada ciego palpaba una parte del animal: pierna, colmillo, trompa, cola, costado, oreja, y daba su versión de lo que entendía por un elefante. Para unos era como una gran columna, para otros como una lanza curva punzante..., sin llegar a saber realmente cómo era este animal.

17 J. Arana, El caos del conocimiento..., op. cit., p. 13. 
Naturaleza y Libertad. Revista de estudios interdisciplinares. Número 3, 2014. ISSN: 2254-9668

Ni siquiera ahí terminan las cosas, porque cada disciplina no se alberga en pabellones inamovibles, sino que continuamente requiere la construcción de ampliaciones y anexos, que se dividen y subdividen sin término. Los matemáticos, por poner un caso, no sólo se sienten desplazados y cohibidos si caen en una reunión de historiadores o meteorólogos, sino que ni siquiera son capaces de reconocerse y sentirse a gusto en su propia casa, cuando se aventuran más allá de las habitaciones que están acostumbrados a transitar. ${ }^{18}$

Está muy bien conocer los árboles, pero también es conveniente saber algo del bosque. El hombre tiene una tendencia natural a buscar el modo de integrar los elementos en un todo. No se satisface con pedazos rotos, quiere saber cómo se unen para tener el plato completo. El siglo XX ha sido testigo de la importancia de la interdisciplinariedad, en el momento histórico del descubrimiento de la dimensión subatómica, no solamente la física se vio afectada, también la química, la biología, las matemáticas... Los descubrimientos de una disciplina no están aislados de los de otras, se trata del mismo edificio aunque se viva en diferentes departamentos o plantas. Juan Arana que ha reflexionado sobre el tema de la interdisciplinariedad y la posibilidad de llevarla a cabo, señala que no es tarea fácil pero es necesaria porque si no logramos aunar los esfuerzos y descubrimientos, será muy difícil afrontar los riesgos que van surgiendo en nuestro mundo y probablemente las propuestas de solución sean ineficaces. Esto nos da pie para presentar la segunda tesis que alertó al mundo científico: la crisis global.

18 Ídem, pp. 14-15. 


\section{EL RECLAMO DE LAS CRISIS GLOBALES Y LA ÉTICA QUE SE CUELA POR LA VENTANA}

Un segundo golpe que recibió el modelo científico moderno fue el colapso de la idea de progreso. La tesis sobre el progreso continuo de las ciencias se ha visto suficientemente refutada con los trágicos acontecimientos bélicos del siglo XX en todo el mundo. La vejación infligida a millones de seres humanos en el continente más avanzado científica y tecnológicamente del mundo echó por tierra la idea de felicidad universal. La carrera armamentística, la guerra fría, la crisis económica y la lucha entre los dos modelos político-económicos de los Estados Unidos y la Unión Soviética acompañaron casi todo el siglo XX. Los descubrimientos científicos y sus aplicaciones tecnológicas también mostraron al mundo su lado más oscuro. $\mathrm{El}$ poder de manipular el átomo enseguida trajo consigo la mayor forma de destrucción masiva de la historia. Lo peor de todo es que aún hay riesgo de que Hiroshima y Auschwitz se repitan, tal vez de manera accidental (Chernobyl, 1986) o tal vez de forma provocada (genocidio de Ruanda, 1994).

Actualmente el poder tecnológico es tal que tenemos la posibilidad de destruir nuestro propio planeta. Las crisis ya no tienen sólo dimensiones locales, sino una dimensión planetaria y prueba de ello son el calentamiento global y los problemas medioambientales. El sueño del mundo próspero y feliz augurado por la ciencia moderna del siglo XIX se convirtió en pesadilla por un factor que no entraba en sus ecuaciones: la capacidad de corrupción del corazón humano. 
Desde luego la ciencia no es mala en sí, es el hombre quien con su obrar realiza actos buenos o malos. $\mathrm{El}$ acto humano es lo imputable éticamente. La ciencia es un tipo de conocimiento que ha ayudado a mejorar la forma de vida de los hombres. Como dice Arana, el problema radica en pretender que este tipo de conocimiento pueda explicarlo todo de forma apodíctica y sólo a partir de su método. Esto se llama reduccionismo porque la realidad es mucho más amplia y compleja de aquello de lo que se puede llegar a comprender solamente desde el paradigma matemático-experimental. Me aventuraría a decir que hoy día ningún científico honrado está en contra de esta afirmación. Pero culturalmente las personas de nuestra sociedad siguen apelando a la ciencia en su búsqueda de respuestas y le piden soluciones que muchas veces ella no puede dar.

El prestigio de la ciencia sigue siendo muy alto, basta con ver cómo en los reportajes de los medios de comunicación la última palabra sobre algún suceso la tienen los científicos y especialistas. Muchas veces estas personas incluso se creen autorizadas para argumentar filosófica o teológicamente sin conocer ninguna de esas disciplinas. Olvidan que ellas también tienen tradiciones seculares que exigen una forma de argumentación y métodos que difieren del científico-experimental. Hablan sin tener conocimientos suficientes y aparte de extralimitarse en sus competencias, confunden a la gente. Aún así, para muchas personas la ciencia se ha convertido en un oráculo, y el enmudecimiento de la filosofía ha contribuido a ello.

También hay indicios de que la filosofía más viva no la elaboran ahora, como hace cien años, los profesores de filosofía, sino individuos que proceden de otros campos y que dan libre curso a sus inquietudes sin respetar las convenciones al uso, que parecen haber sido 
Dossier crítico sobre el libro: Los sótanos del universo

diseñadas para atar bien corto a quien las acate. Aunque no sienten cátedra en departamentos y facultades, ni sean aún objeto de tesis doctorales o ediciones críticas, no por ello dejan de hacer oír su voz: publican los únicos libros que se venden y suscitan casi todos los debates que llegan al público. Los sabios oficiales no dejan de censurarles, pero eso es algo que no llega a inquietarles ni poco ni mucho. [...] El fenómeno ha adquirido tal notoriedad que ya ha recibido el interesado (desde el punto de vista comercial) apelativo de "tercera cultura" (Brockman, 1996). Las firmas con mayor éxito son, desde luego, las de científicos de renombre dispuestos a ocupar con desenfado este terreno que la incuria de los académicos ha dejado prácticamente virgen. ${ }^{19}$

Este prestigio y la fuerte presencia de la ciencia en la sociedad en general no se debe a que los no-especialistas comprendan cabalmente las tesis descubiertas o los complejos cálculos elaborados para llegar a resultados favorables, sino sobre todo al hecho de que sus efectos se ven en la vida cotidiana. La mayoría no conoce las bases físicas de las ondas hertzianas, ni tampoco les interesa, pero están encantados escuchando sus programas de radio. Ni qué decir de la televisión, Internet, los dispositivos móviles, las aplicaciones del efecto fotoeléctrico, etc. Es decir, en gran parte la razón del éxito de la ciencia proviene del aval que le da la tecnología, ya que la tecnología es la aplicación práctica de la ciencia.

Me parece conveniente considerar aunque sea brevemente en el tema tecnológico. La gran revolución científica estuvo acompañada por una gran revolución tecnológica. Hoy día esto resulta patente. La tecnología no solo ha cambiado nuestro modo de vivir sino que lo ha condicionado. $Y$ aunque éste no es el lugar para detenerse a estudiar más a fondo la relación ciencia-

19 J. Arana, Los sótanos del universo..., op. cit., p. 46. 
tecnología, al menos mencionamos su maridaje. La Historia de la Ciencia nos muestra su mutua influencia, a veces la técnica impulsa la ciencia y otras es al revés. Ya en el proyecto baconiano se postulaba la idea de avanzar en el conocimiento de la naturaleza para mejorar la condición humana. No se trataba del saber por el saber, sino que el saber había que aplicarlo en el mejoramiento del modo de vida de la humanidad.

Lo que sucedió fue que el conocimiento cada vez mayor de la naturaleza condujo a su control y también a su abuso. La Revolución Industrial se llevó a cabo tras haber descubierto los procesos de automatización y producción en serie. Esto trajo muchos beneficios en cuanto a la calidad de vida de la sociedad, pero también trajo los efectos colaterales negativos que conocemos y que nos siguen afectando, aquí no me refiero al ya conocido problema social, sino al daño ambiental.

Desde hace varias décadas se ha podido comprobar una serie de problemas medioambientales regionales y globales. Los gobiernos han intentado resolverlos apelando a los conocimientos técnicos y científicos, pero se han dado cuenta de que este es sólo un aspecto del problema. Además de las respuestas que ofrecen la ciencia y la tecnología, hace falta que también se involucren otro tipo de disciplinas no científicas para atender sobre todo las cuestiones de índole moral. Y es que la ética actúa de forma transversal en todas las disciplinas: la economía, la política, el derecho...

A continuación presentaré muy sintéticamente el caso del Cambio Climático como ejemplo de problema al que se ha llegado sobre todo por causas tecnológicas y cómo la interdisciplinariedad de las ciencias ha sido un reclamo necesario para encontrar soluciones. Interdisciplinariedad en la que también se ha visto envuelta la filosofía a través de la ética. 
En 1965 en la ciudad de Boulder (USA) climatólogos y científicos de distintas disciplinas asistieron a una conferencia sobre las "Causas del Cambio Climático". Uno de los asistentes fue Edward Lorenz, que entonces trabajaba en el MIT. En su discurso inaugural explicó que las más leves modificaciones en las condiciones iniciales podían provocar un enorme cambio aleatorio en el clima posterior (luego esto se conoció bajo el nombre de "efecto mariposa"). Sus palabras de que el clima podía ser o no determinista y de que era probable que nunca lo supiéramos fue impactante. Tuvieron que admitir que el cambio climático se debía a un conjunto complejo de causas y que los medios de investigación tradicionales no ayudaban a conocerlo:

\footnotetext{
Este cambio de perspectiva hacia lo que algunos calificaron de planteamiento holístico impulsó a los científicos a modificar sus métodos de trabajo. No se podía construir un modelo verosímil de cambio climático, por no hablar de cotejarlo con los datos reales, sin tener información sobre muchas cosas distintas. Estaba siendo dolorosamente claro que los científicos de las diversas disciplinas se necesitaban mutuamente. El congreso de Boulder de 1965 fue sólo una de las muchas ocasiones en las que diferentes tipos de especialistas comenzaron a interactuar más estrechamente apoyándose en los hallazgos de los demás o, con una actitud igualmente valiosa, poniéndolos en cuestión. ${ }^{20}$
}

Pero no solamente fue necesaria la participación de las disciplinas científicas en general, sino también de la filosofía. Lo curioso ha sido la manera natural como ella se introdujo en la problemática ambiental. La filosofía, a quien se le había cerrado la puerta, ha podido entrar nuevamente por la ven2006, p. 83. 
tana. La ventana de la ética o reflexión filosófica práctica acerca del adecuado comportamiento humano. Ocurrió que en el día de la Tierra de 1970 en la ciudad de San Francisco, por primera vez de forma explícita los científicos pidieron a los filósofos que se involucraran en los problemas ambientales para buscar los fundamentos éticos que pudieran resguardar los abusos cometidos en la sociedad. A partir de ese día se funda una nueva disciplina con carácter académico: The Environmental Ethics. ${ }^{21}$

Insisto, uno de los temas centrales de nuestro tiempo es la reflexión ética de la investigación y resultados científicos. Aquellas moralejas de los pensadores y literatos que advertían del uso irresponsable del conocimiento científico, como por ejemplo el Frankenstein de Mary Shelley, ya no resulta tan ingenuo. Sin ánimo de ser catastrofista, hoy día se cuenta con conocimientos y tecnología que pueden determinar el futuro del género humano y del resto del planeta.

No se trata de vivir con miedo, sino con sentido de responsabilidad. Las palabras de Pierre Curie en su discurso de agradecimiento por el Premio Nóbel pronunciado en 1905 me parecen pertinentes:

Se puede imaginar que en manos criminales el radio pueda hacerse muy peligroso, y en este punto nos podemos preguntar si la humanidad extrae ventajas conociendo los secretos de la naturaleza, si está madura para beneficiarse o si este conocimiento no le resultará perjudicial. El ejemplo de los descubrimientos de Nóbel es característico: los poderosos explosivos han permitido a los hombres llevar a cabo trabajos admirables. También son un medio terrible de destrucción en las manos de grandes criminales que arrastran a los pue-

21 Cf. http://www.cep.unt.edu/novice.html Última visita: 11-Enero-2013. 
blos a la guerra. Yo soy de los que piensan con Nóbel que la humanidad extraerá más bien que mal de los nuevos descubrimientos ${ }^{22}$.

Hemos visto cómo la insuficiencia de la mecánica clásica y la falacia de la idea de progreso han socavado el modelo de certidumbre científica que campeó la historia de la ciencia desde el Renacimiento. ¿Y ahora qué? La propuesta de Arana es un nuevo giro epistemológico que se denomina: epistemología del riesgo.

\section{EPISTEMOLOGÍA DEL RIESGO Y ANTROPOLOGÍA DEL}

\section{CONOCIMIENTO}

Las nuevas tecnologías han hecho accesibles innumerables conocimientos. Casi todo está en Internet, sólo hay que saber cómo buscarlo. Cuando se trata de conocimientos técnicos la información es precisa y especializada, cuando se trata de conocimientos científicos un poco menos, pero todavía pueden encontrarse artículos o "blogs" de autores con rigor académico que nos ayudan a encontrar respuestas.

Sin embargo, a medida que vamos saliendo de los reductos especializados y pretendemos integrar los conocimientos es cuando comienzan los problemas serios. Podemos encontrar auténticas contradicciones que nos generan dudas. Es entonces cuando entramos en el reino de las opiniones (la doxa), la

22 J. M. Sánchez Ron, Marie Curie y su tiempo, Barcelona, Ediciones Folio, 2003, p. 117. Menciona como fuente: P. Curie, “Conférence Nobel”, en: Les Prix Nobel en 1903, Estocolmo, Imprimerie Royale, P. A. Norsted \& Döner, 1912, pp. 1-7. 
situación de imperfección más temida por los científicos y los filósofos. En el terreno de la opinión todo vale, es campo abonado para el relativismo y el escepticismo (dos grandes corrupciones del conocimiento verdadero).

Como decía, uno de los grandes problemas del conocimiento consiste en integrar las verdades que se hallan esparcidas en los centenares de reductos especializados. La tarea más ardua es lograr "la gran unificación”, o al menos "la gran relación de puntos" (connecting dots). La posmodernidad está en contra de los "súper sistemas" holísticos, que intentan explicarlo todo. En esto último yo también me apunto sin caer en el pensiero debole. La idea no consiste en construir un "súper sistema"; me parece que en el gran puzzle de la realidad hay zonas que se pueden ir completando poco a poco. Se trata de ir uniendo piezas sueltas para formar cada figura que compone el gran puzzle y que da sentido a una obra mayor que anhelamos comprender. Al unir adecuadamente esas figuras se irá comprendiendo mejor el tema final, pero la labor debe ser cooperativa, no individual o sectorial. Para ello hace falta salir del "reducto", tomar distancia de las fichas individuales y mirar lo que han conseguido los demás para encontrar la forma de integrarlas unas con otras. Hace falta una mirada más amplia y colaborativa. Es el principal argumento para fomentar la interdisciplinariedad. Las ciencias positivas se han atomizado tanto que la tarea es complicada. Muchos miran con pesimismo incluso el futuro de la propia ciencia.

Para algunos autores, en sentido epistemológico, la ciencia vive momentos duros. Es la tecnología la que está en auge. En efecto, la tecnología ha obrado y obra maravillas. Se puede decir que por ella nuestro mundo se ha "empequeñecido" y de alguna manera se ha vuelto "abarcable". La tecnología ha hecho que el tiempo del actuar y vivir se acelere, podemos 
estar al otro extremo del mundo en cuestión de horas, en cuestión de segundos podemos tener la información que antes tardábamos meses en encontrar. Uno puede escribir una palabra en el buscador de un ordenador y no solamente aparecen millones (literalmente) de resultados sino que el tiempo de respuesta se computa en segundos y décimas de segundo. La vida se presenta más que nunca como un frenesí. Esto hace que las cosas parezcan más fáciles, pero en realidad son más difíciles. ¿No sería todo esto cierto efecto "pantalla" que nos produce el éxito de la tecnología? ¿Es cierto que la tecnología ha logrado protagonismo pero la ciencia está de capa caída? ¿Qué dicen estos preconizadores del fin de la ciencia?

Arana nos explica esta situación al introducir las teorías de la complejidad. Tratemos este último punto para terminar estas reflexiones.

\footnotetext{
Algunos definen la ciencia como el arte de las verdades fáciles. Por simetría, debería decirse que la filosofía lo es de las verdades difíciles. Esta simplista división tiene la ventaja de explicar por qué algunas preguntas que formaban parte de la filosofía (ya que no se sabía muy bien cómo resolverlas) le fueron sustraídas en cuanto aparecieron respuestas merecedoras de general aprobación. El establecimiento de nuevos métodos ha tenido casi siempre la virtud de convertir en cientificas cuestiones que antes eran filosóficas, en la misma proporción que facilitaron el acceso a ellas y disiparon su dificultad. ${ }^{23}$
}

Si se dan por válidas estas definiciones, la filosofía podría volver a recobrar un protagonismo que no ha tenido en los últimos siglos. Si hemos llegado a un punto en el que las preguntas se complican de tal manera que ya 
no hay respuestas fáciles y la ciencia tiene poco que decir, la ciencia podría llegar a su fin. Esto me cuesta aceptarlo, la ciencia no llegará a su fin, aunque algunos autores han hecho análisis muy aventurados a este respecto, como J. Horgan o L. Smolin ${ }^{24}$. En el libro de Arana hay una cita de este último autor que es muy pesimista:

Pertenezco a la primera generación de físicos formados tras la formulación del modelo estándar de la física de partículas. Cuando me reúno con viejos amigos y antiguos compañeros de la universidad, a veces nos preguntamos: “¿Qué hemos descubierto de lo que nuestra generación pueda sentirse orgullosa?". Si hablamos de descubrimientos fundamentales, establecidos mediante la experimentación y explicados por una teoría, descubrimientos del nivel de los mencionados hasta el momento, la respuesta es, muy a nuestro pesar: “¡Nada!”. (Smolin, 2007:14). ${ }^{25}$

Más adelante L. Smolin reconoce que para el progreso de la ciencia es conveniente enfrentarse de nuevo a las preguntas más profundas sobre el tiempo y el espacio, la teoría cuántica y la cosmología. Es decir, replantearse las cuestiones fundamentales. Arana señala que para algunos un ritmo desbocado de progresión es síntoma infalible de la inminencia de un parón. Su punto de vista es que la filosofía y la ciencia deberían ir de la mano, en lugar de pretender suplantarse y excluirse mutuamente. Los descubrimientos del siglo XX han demostrado suficientemente que no hay temas simples, sino

24 Cf. J. Horgan, El fin de la ciencia. Los limites del conocimiento en el declive de la era científica, Barcelona, Paidós, 1998; L. Smolin, Las dudas de la física en el siglo XXI. ¿Es la teoría de cuerdas un callejón sin salida?, Barcelona, Crítica, 2007. J. Arana menciona a estos autores en su obra. 25 J. Arana, Los sótanos del universo, op. cit., p. 176. 
temas tratados de forma reductiva. Ahora se apela a la consideración de esa realidad complicada y difícil.

Dos disciplinas que hoy día están requiriendo esa investigación de los fundamentos son la genética y las neurociencias. En estas áreas confluyen muchas disciplinas científicas y ni qué decir filosóficas. La interdisciplinariedad es obligada, como veíamos en el caso de la ecología, irrenunciable diría yo. Es muy claro deducir que de lo contrario se caería en un reduccionismo epistemológico. La pregunta ya no es si es necesaria la interdisciplinariedad; sino cómo podemos trabajar de manera interdisciplinaria. La respuesta no está escrita, hay que descubrirla sobre la marcha. Personalmente me llama positivamente la atención la manera de trabajar en los centros de investigación de los Estados Unidos, donde colaboran científicos, filósofos e incluso teólogos en algunas universidades. En España todavía hay mucho camino por recorrer, pero el tiempo irá mostrando que se trata de un tema obligado.

La epistemología del riesgo supone la posibilidad de equivocarse, pero esta posibilidad siempre ha estado en la investigación científica. Lo que subraya es la necesidad de evitar la ceguera de buscar lo que dada nuestra condición humana jamás podremos alcanzar: el saber absoluto y apodíctico. La epistemología del riesgo es un llamado a una adecuada antropología. No somos una inteligencia que conoce, sino hombres que conocen gracias a su inteligencia. La antropología del conocimiento admite diversas fuentes y formas de aproximación, no solamente el método científico-experimental. Esto no implica contentarse con vaguedades o medianías, sino contentarse con lo que hemos alcanzado a través de nuestro mayor esfuerzo. Esta epistemología es humana porque admite nuestras limitaciones y espolea para que esas limitaciones puedan superarse poco a poco, sabiendo que más adelante 
puede haber otra manera mejor de explicar la realidad. Es preciso recordar que una cosa es lo que se conoce (de ello trata la gnoseología) y otra cosa es la realidad que se conoce (la ontología); y que ésta es siempre mucho más rica que aquella y por tanto, imposible de ser agotada.

\begin{abstract}
Es muy relevante mostrar que ni Hume, ni Kant, ni la epistemología positivista o neopositivista han conseguido aportar una fundamentación incuestionable de la causalidad sobre bases no ontológicas. Constituye una prueba fehaciente de que el giro copernicano introducido por Kant en la teoría del conocimiento es un fiasco. Ni más ni menos. Lo cual a su vez abre la puerta a una reconsideración del trasfondo ontológico de la causalidad desde una epistemología del riesgo: la presunción de que hay entidades objetivas tras las intuiciones sensibles y conexiones genéticas entre ellas no es más problemática que la que solo admite un simple juego de representaciones. Hay además indicios complementarios para convertirla en una creencia racional, empezando por los innegables éxitos predictivos de la ciencia. ${ }^{26}$
\end{abstract}

\title{
6. CONCLUSIÓN
}

La mecánica cuántica ha obligado a cambiar las reglas del juego. Esta vez desde la misma ciencia se ha demostrado que el mecanicismo no tiene los recursos necesarios para explicar la compleja realidad que nos rodea. Al contrario, mediante sus métodos reduccionistas pretende justificar los distintos hechos de la naturaleza, cuando lo que hace realmente es una descripción basta y superficial que resulta completamente anacrónica hoy día. Sin lugar a dudas tiene sus seguidores y los seguirá teniendo porque ofrece respuestas "de 26 Ídem, pp. 372-373. El subrayado es de la fuente. 
brocha gorda”. Lo que pasa es que es insuficiente. Tanto el macro como el microcosmos han revelado aspectos de complejidad que aún hoy no sabemos cómo abordar.

La ciencia actual todavía va a tientas y a cada paso surgen noticias inesperadas de descubrimientos que no están suficientemente demostrados o son erróneos (la supuesta detección del Bosón de Higgs, o que los neutrinos puedan ser más rápidos que los fotones). ¿Será esto una señal de precipitación?, ¿de que no atinamos con las respuestas adecuadas? De cualquier manera "el mundo gira y la nave va", la nave tecnológica no se detiene y sigue transformando nuestras vidas a velocidades que apenas podemos asumir.

Entre las "nuevas" opciones de investigación surge de nuevo el "antiguo" método filosófico, que no tiene miedo de nadar en aguas peligrosas, que siempre parece a punto de ahogarse pero vuelve a surgir una y otra vez. La filosofía no es un conocimiento exacto, y mucho menos perfecto, se trata de un conocimiento de características humanas que incluye deseo y afecto (Philo + sophos). La ventaja que tiene es que está abierta a todo tipo de aportaciones de otras áreas con la sola condición de que sean lógicas y razonables. Incluso se atreve a buscar una pizca de razonabilidad en lo ilógico, por esa sed de conocimiento que es innata en él.

La antigua frase de Aristóteles: "todos los hombres por naturaleza desean saber" sigue brillando en el horizonte de nuestras vidas y lo que Arana sugiere es que no estrechemos las miras sólo por razones de método. Seamos rigurosos pero no hasta el punto de caer en la escrupulosidad que supone la certidumbre total. El conocimiento perfecto no casa con las imperfección humana. La auténtica antropología del conocimiento es la que asume la limitación humana sin caer en el escepticismo que supone la derrota de la 52 
inteligencia. La imagen de Agustín de Hipona jugando en la playa, intentando llenar el hoyo en la arena con su pequeño cubo cargado de agua del mar, sigue siendo una metáfora de nuestra condición.

La tesis de Juan Arana de que Ciencia y Filosofía deben darse la mano significa una vuelta a la búsqueda del saber completo, esto supone sobre todo el rechazo de los reduccionismos. En realidad, ambas disciplinas apuntan en la misma dirección e incluso hay puntos posibles de encuentro. Por ejemplo, esto se ve claramente en "la etapa del descubrimiento", que es la etapa previa a la formulación de la hipótesis inicial donde el investigador se deja atrapar por la curiosidad, las intuiciones personales y un fuerte impulso para buscar respuestas.

¿Cómo es posible la interdisciplinariedad? Actualmente se ensayan numerosos métodos de investigación, una buena señal. Pero se trata de una pregunta abierta que tiene múltiples respuestas y hasta que no comencemos a practicarla no sabremos cuál es la que más nos conviene. Un buen punto de partida puede ser la revisión de los conceptos y terminologías o como dice Arana: “...un «programa de desarme lingüístico y metodológico» de las ciencias, como punto de partida y puerta de acceso a otro programa más ambicioso de «síntesis teórica»"27.

Me parece que también es importante crear un buen ambiente de investigación, me refiero sobre todo al clima de respeto cordial en el trato y tono humano del grupo multidisciplinar. Resulta conveniente salir de nues-

27 J. Arana, El caos del conocimiento..., op. cit., p. 21. A continuación enumera una serie de diez puntos que pueden orientar el emprendimiento multidisciplinar, y admite que puede ser una utopía, pero por algo hay que comenzar. 
tros parapetos y mostrar sin rubor lo que sabemos -y lo que no sabemos-. Muchas veces se olvida que una adecuada camaradería facilita la apertura de la mente al conocimiento y a la comprensión de puntos de vista diferentes. Desde luego mucho más que enzarzarse en una especie de competencia con técnicas a lo Sun Tzu donde la envidia y la ambición propia minan las relaciones del equipo.

El otro paso difícil consiste en realizar el esfuerzo necesario para aprender lo suficiente de las demás disciplinas, lo cual facilitará la comprensión y permitirá diálogos razonables de los temas desde distintas perspectivas. El filósofo debe tener nociones científicas e interesarse por ellas y el científico debe conocer el método filosófico y la lógica que impera en las reflexiones metafísicas. Como se puede ver, esto requiere tiempo y constancia, notas que no son extrañas en los descubrimientos que han surgido a lo largo de la Historia de la Ciencia pero que son auténticos desafíos para los grupos de investigación espoleados por mostrar resultados y cumplir las papeletas requeridas por las agencias oficiales de evaluación.

La premisa principal consiste en no cerrarnos puertas por una estrechez metodológica, cayendo en reduccionismos que dan un aparente poder sobre el mundo natural. El mundo natural va mucho más allá de la dimensión de nuestros sentidos orgánicos y físicos. De nuevo se trata de profundizar en la ontología y la gnoseología para atinar con la adecuada epistemología, estando abiertos al riesgo.

En este sentido, una de tantas lecciones que ofrece Juan Arana en su libro Los sótanos del universo es que: 
Naturaleza y Libertad. Revista de estudios interdisciplinares. Número 3, 2014. ISSN: 2254-9668

La dualidad onto-gnoseológica que he apuntado al comienzo, no es un simple dato, sino que constituye también un primer desafío teórico. No basta con distinguir, a lo Kant o a lo Schopenhauer, el plano de lo nouménico y el fenoménico, el mundo de la representación y el de lo en si. Cabe postular que los hechos empíricos, con los conceptos y principios que utilizamos para ordenarlos, no sirven para descubrir y conocer la realidad, que solo cabría rozar o asir por otros procedimientos teóricos o prácticos. Pero los que han hecho la opción metódica de valerse exclusivamente de hechos, conceptos y principios como medios válidos de conocimiento, por fuerza verán en ellos la única puerta de acceso a la realidad, no importa lo estrecha u obscura que a tal fin resulte. $Y$ si se les dice que así no se accede a la realidad sino a otra cosa, entonces empezarán a considerar la "realidad" como una lejana quimera. Una de las condiciones de posibilidad para que el diálogo ciencia-filosofia resulte fructífero es que el filósofo acepte que, en efecto, a través de la ciencia se accede a la realidad, aunque él no se limite a buscarla mediante la conceptuación y ordenación de la experiencia, como hace el científico. ${ }^{28}$

Los sótanos del universo es un compendio de los principales temas de la Filosofía de la Naturaleza y Juan Arana ha procurado alcanzar el equilibrio necesario para no caer en simplismos y al mismo tiempo señalar con argumentos de peso los puntos nucleares de las aproximaciones filosóficas y científicas que han prevalecido en la Historia de la Ciencia y que hoy día ocupan la mente de los investigadores. Debo agradecerle este ambicioso trabajo que, gracias a su cosmovisión, ayuda a orientar nuestros estudios y reflexiones. Vaya para él mi más sincera enhorabuena.

Miguel Acosta

macosta@ceu.es

28 J. Arana, Los sótanos del universo..., op. cit., p. 247. El subrayado es de la fuente. 
